\title{
X-Ray Diffraction, Mössbauer Spectroscopy, and Magnetoelectric Effect Studies of Multiferroic $\mathrm{Bi}_{5} \mathrm{Ti}_{3} \mathrm{FeO}_{15}$ Ceramics
}

\author{
T. Pikula ${ }^{a, *}$, P. GuzdeK ${ }^{b}$, J. DzIK $^{c}$, A. Lisinska-CzeKaJ ${ }^{c}$ And E. JARTYCH ${ }^{a}$ \\ ${ }^{a}$ Lublin University of Technology, Institute of Electronics and Information Technology, \\ Nadbystrzycka 38A, 20-618 Lublin, Poland \\ ${ }^{b}$ Institute of Electron Technology, Cracow Division, Zabłocie 39, 30-701 Kraków, Poland \\ ${ }^{c}$ University of Silesia, Department of Materials Science, Śnieżna 2, 41-200 Sosnowiec, Poland
}

\begin{abstract}
$\mathrm{Bi}_{5} \mathrm{Ti}_{3} \mathrm{FeO}_{15}$ ceramics belongs to multiferroic class of materials. In this work it was prepared by solidstate sintering method and investigated by X-ray diffraction, Mössbauer spectroscopy, and magnetoelectric effect measurements. As it was proved by X-ray diffraction studies the single-phase $\mathrm{Bi}_{5} \mathrm{Ti}_{3} \mathrm{FeO}_{15}$ compound was obtained. The Mössbauer investigations revealed paramagnetic character of the compound at room temperature as well as at $80 \mathrm{~K}$. Magnetoelectric measurements were carried out at room temperature using lock-in dynamic method and they proved presence of magnetoelectric coupling in this material. Additional magnetoelectric studies were carried out after subsequent electric poling of the sample. It was found that the maximum value of the coupling coefficient was almost twice bigger than in the case without the initial poling and reached a value of $\alpha_{M E} \approx 20.7 \mathrm{mV} \mathrm{cm}{ }^{-1} \mathrm{Oe}^{-1}$.
\end{abstract}

DOI: 10.12693/APhysPolA.127.296

PACS: 75.85.+t, 85.80.Jm, 61.05.cp, 76.80.+y

\section{Introduction}

Multiferroics are the materials which exhibit at least two ferroic states simultaneously. Especially interesting is the group of magnetoelectrics in which both ferroelectric and magnetic ordering coexist. They exhibit magnetoelectric coupling which makes possible induction of magnetization by applying external electric field or induction of electric polarization by applying external magnetic field [1]. A possibility to control electric properties by magnetic field and magnetic properties by electric field in multifferroic materials attracts a lot of interest due to their potential applications in sensor technique, microelectronics, energy harvesting and spintronics $[1,2]$. The most frequently investigated multiferroic compound is $\mathrm{BiFeO}_{3}$. It offers ferroelectric and antiferromagnetic ordering at relatively high temperatures [3]. However, the existence of spin cycloid in this compound averages out a linear magnetoelectric coupling [4].

$\mathrm{Bi}_{5} \mathrm{Ti}_{3} \mathrm{FeO}_{15}$ ceramics belongs to the family of the $\mathrm{Au}-$ rivillius compounds. It has layered structure which is built of four perovskite-like slabs of nominal composition $\left(\mathrm{Bi}_{3} \mathrm{Ti}_{3} \mathrm{FeO}_{13}\right)^{2-}$ sandwiched by fluorite-like $\left(\mathrm{Bi}_{2} \mathrm{O}_{2}\right)^{2+}$ layers [5-8]. The so-called four layered compound exhibit ferroelectricity with the Curie temperature $T_{\mathrm{C}}=730^{\circ} \mathrm{C}$ and antiferromagnetic ordering, however, there is no agreement to the value of the Néel temperature in reported data $[9,10]$. From the other hand $\mathrm{Bi}_{5} \mathrm{Ti}_{3} \mathrm{FeO}_{15}$

*corresponding author; e-mail: t.pikula@pollub.pl can be viewed as an atomic stacking of the ferroelectric $\mathrm{Bi}_{4} \mathrm{Ti}_{3} \mathrm{O}_{12}$ compound and the well-known $\mathrm{BiFeO}_{3}$ multiferroic, so it is supposed to offer magnetoelectric coupling [5]. In fact, magnetoelectric properties of the $\mathrm{Bi}_{5} \mathrm{Ti}_{3} \mathrm{FeO}_{15}$ compound have already been investigated [10, 11-13]. The authors of the papers [11] and [12] have reported maximum value of magnetoelectric coupling coefficient $\alpha_{\mathrm{ME}}$ equal to $17 \mathrm{mV} \mathrm{cm}^{-1} \mathrm{Oe}^{-1}$. The measurements were performed using static method and the sample was initially poled electrically and magnetically. In the work [10] investigations of $\alpha_{\mathrm{ME}}$ at room as well as at liquid nitrogen temperatures have been performed. The obtained maximum values were 0.1 and $2.8 \mathrm{mV} \mathrm{cm}^{-1} \mathrm{Oe}^{-1}$, respectively. In turn, the authors of the paper [13] have revealed maximum value of $\alpha_{\mathrm{ME}}$ equal to $8.28 \mathrm{mV} \mathrm{cm}^{-1} \mathrm{Oe}^{-1}$. In the light of such discrepancies there is a necessity of re-examination of magnetoelectric properties of the $\mathrm{Bi}_{5} \mathrm{Ti}_{3} \mathrm{FeO}_{15}$ ceramics and this is the main aim of the present paper.

\section{Experiment}

$\mathrm{Bi}_{5} \mathrm{Ti}_{3} \mathrm{FeO}_{15}$ compound was prepared by mixed oxide method. High purity oxide powders were mixed according to the reaction

$$
5 \mathrm{Bi}_{2} \mathrm{O}_{3}+6 \mathrm{TiO}_{2}+\mathrm{Fe}_{2} \mathrm{O}_{3} \rightarrow 2 \mathrm{Bi}_{5} \mathrm{Ti}_{3} \mathrm{FeO}_{15}
$$

and subjected to grinding in a ball mill for $24 \mathrm{~h}$. After that the powder was pressed and formed into small disks under pressure of $30 \mathrm{MPa}$. The synthesis was carried out in air at $750{ }^{\circ} \mathrm{C}$ for $10 \mathrm{~h}$. The synthesized material was milled in a wet medium; next it was dried, pressed and formed into pellets under pressure of $60 \mathrm{MPa}$. The sintering was performed in air at temperature $980^{\circ} \mathrm{C}$ for $3 \mathrm{~h}$. 
Phase composition and structure of the $\mathrm{Bi}_{5} \mathrm{Ti}_{3} \mathrm{FeO}_{15}$ sample were investigated by X-ray diffraction (XRD) with Empyrean PANalytical diffractometer using $\mathrm{Cu} K_{\alpha}$ radiation. X'Pert HighScore Plus computer program equipped with the ICDD PDF2 data base was used to phase analysis and the Rietveld refinement of the structure.

The Mössbauer spectra were registered using POLON spectrometer which was working in transmission geometry and constant acceleration mode.

The room temperature magnetoelectric (ME) measurements were performed using dynamic lock-in method. The pellets of $\mathrm{Bi}_{5} \mathrm{Ti}_{3} \mathrm{FeO}_{15}$ compound were placed into a static DC magnetic field $H_{\mathrm{DC}}$ with superimposed small sinusoidal AC magnetic field $H_{\mathrm{AC}}$. Both magnetic field directions were perpendicular to the sample surface and they were measured by a Hall probe SM 102. The ME output voltage $V_{\text {out }}$ was measured by SR830 lock-in amplifier. The first order ME coupling coefficient for a sample of thickness $d$ was calculated using formula

$$
\alpha_{\mathrm{ME}}=\frac{1}{d} \frac{V_{\mathrm{out}}}{H_{\mathrm{AC}}} .
$$

Additional measurements of $\alpha_{\mathrm{ME}}$ were carried out after initial poling of the $\mathrm{Bi}_{5} \mathrm{Ti}_{3} \mathrm{FeO}_{15}$ compound. The sample was electrically poled at $110^{\circ} \mathrm{C}$ under the field $2 \mathrm{kV} \mathrm{mm}{ }^{-1}$ within $2 \mathrm{~h}$. After that the sample was cooled down to room temperature in a presence of the electric field within $12 \mathrm{~h}$.

\section{Results and discussion}

The results of XRD measurements are presented in Fig. 1. The angular position of diffraction lines agree well with positions for orthorhombic $\mathrm{Bi}_{5} \mathrm{Ti}_{3} \mathrm{FeO}_{15}$ phase given in ICDD PDF 01-82-0063 card. No other diffraction peaks except for that of the desirable compound can be detected, indicating the formation of the single $\mathrm{Au}$ rivillius phase. Numerical fitting by the Rietveld method was carried out under an assumption of Fmm2 (no. 42) space group. The calculated lattice parameters are as follows: $a=5.467(2) \AA, b=5.439(1) \AA$ and $c=41.18(1) \AA$. They are in good agreement with the data reported by other authors $[5,6,14]$.

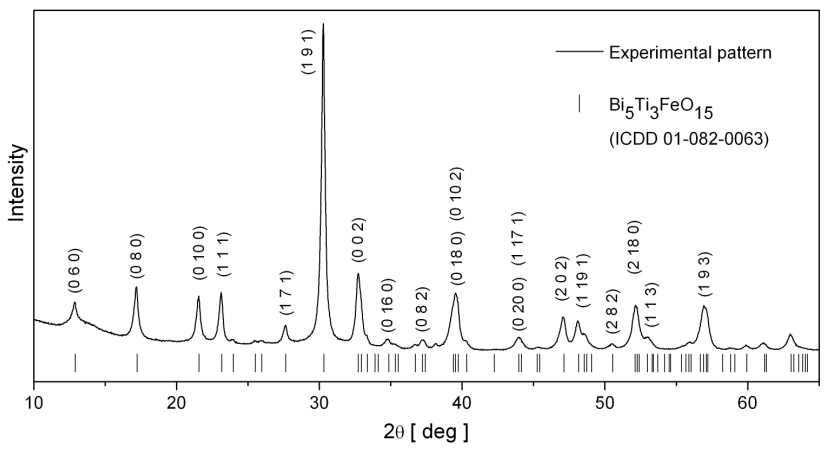

Fig. 1. X-ray diffraction pattern for $\mathrm{Bi}_{5} \mathrm{Ti}_{3} \mathrm{FeO}_{15}$ compound.
Figure 2 presents the Mössbauer spectra for $\mathrm{Bi}_{5} \mathrm{Ti}_{3} \mathrm{FeO}_{15}$ registered at room temperature (RT) and liquid nitrogen (LN) temperature. In both cases they are doublets which proves paramagnetic character of the sample at least down to $80 \mathrm{~K}$. Moreover, our earlier investigations [9] have shown that there is no indication of any magnetic transition down to $2 \mathrm{~K}$. However, the obtained negative value of the Curie-Weiss temperature is indicative for antiferromagnetic interaction [9]. It is worth noting that there are no traces of other components in the Mössbauer spectra. This confirms XRD results that single-phase compound was obtained. In order to reveal the details of Mössbauer spectrum additional RT measurement at lower range of velocity was performed (Fig. 3). The best fit for the experimental data was obtained using two doublets as it is shown in Fig. 3. Their hyperfine interactions parameters are presented in Table and they are almost identical to those reported in [15] and close to those given in [8].

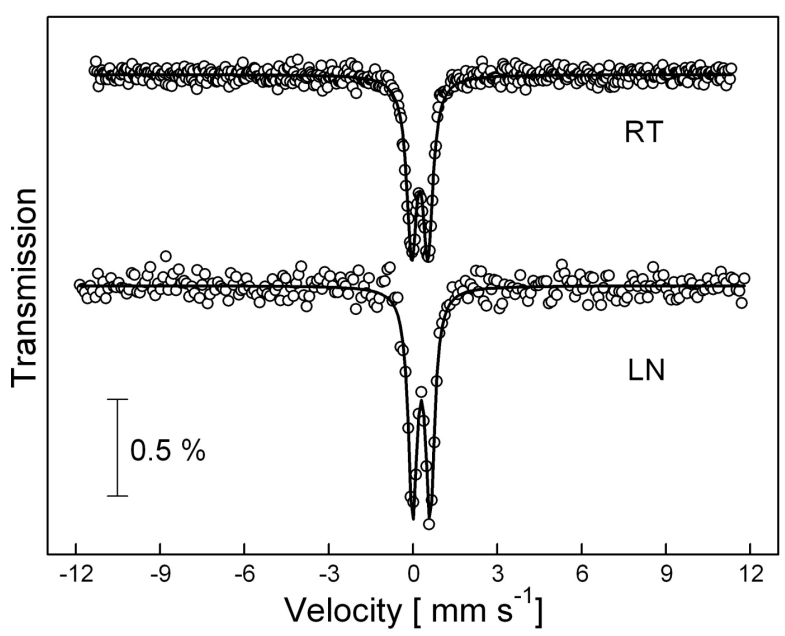

Fig. 2. Mössbauer spectra for $\mathrm{Bi}_{5} \mathrm{Ti}_{3} \mathrm{FeO}_{15}$ compound registered at room temperature (RT) and liquid nitrogen temperature $(80 \mathrm{~K})$.

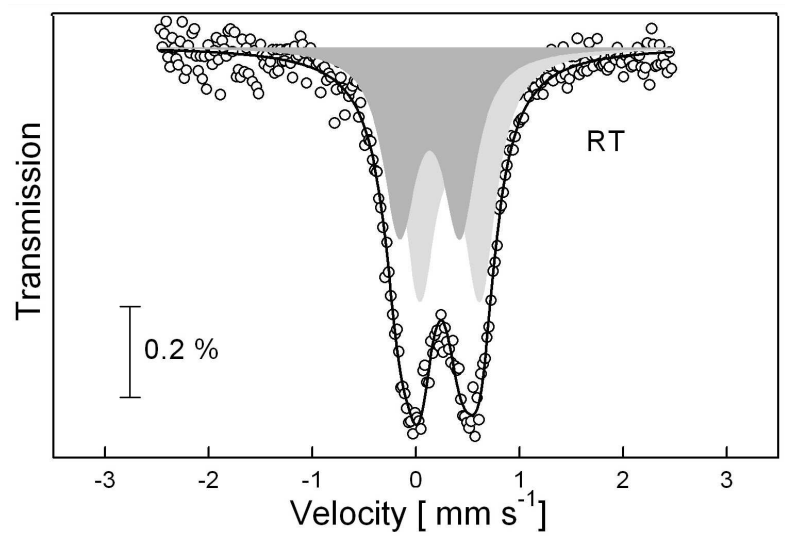

Fig. 3. Fitted room-temperature Mössbauer spectrum for $\mathrm{Bi}_{5} \mathrm{Ti}_{3} \mathrm{FeO}_{15}$ registered at lower range of velocity. 
TABLE

Hyperfine interactions parameters of $\mathrm{Bi}_{5} \mathrm{Ti}_{3} \mathrm{FeO}_{15}$ derived from RT Mössbauer spectra: $\delta$ - isomer shift relative to $\alpha$-Fe standard, $\Delta-$ quadrupole splitting, $\Gamma-$ half width at half maximum of spectral lines, $\chi^{2}$ - fitting parameter, $A$ - relative area of the component; uncertainty for $\delta, \Delta$ and $\Gamma$ is $0.01 \mathrm{~mm} \mathrm{~s}^{-1}$

\begin{tabular}{c|c|c|c|c|c}
\hline \hline $\begin{array}{c}\text { Com- } \\
\text { ponent }\end{array}$ & $\begin{array}{c}\delta \\
{\left[\mathrm{mm} \mathrm{s}^{-1}\right]}\end{array}$ & $\begin{array}{c}\Delta \\
{\left[\mathrm{mm} \mathrm{s}^{1}\right]}\end{array}$ & $\begin{array}{c}\Gamma \\
{\left[\mathrm{mm} \mathrm{s}^{-1}\right]}\end{array}$ & $\chi^{2}$ & $\begin{array}{c}A \\
{[\%]}\end{array}$ \\
\hline Doub.1 & 0.43 & 0.58 & 0.18 & 1.26 & 57 \\
Doub.2 & 0.24 & 0.58 & 0.18 & & 43
\end{tabular}

It may be noted that the values of quadruple splitting, $\Delta$, for both doublets are equal within the limit of experimental error while their isomer shifts, $\delta$, differ significantly. The isomer shift of nuclear levels is related to the density of $s$-type electrons at the site of ${ }^{57} \mathrm{Fe}$ nucleus. Therefore, the value depends on both the valence state of iron and its coordination. The values of $\delta$ determined from the fitted subspectra are typical for $\mathrm{Fe}^{3+}$ ions.

As it was shown in $[8,9]$ the iron ions substitute Ti ions inside the oxygen octahedra. Two doublets applied for fitting the spectra in Fig. 3 reflect two nonequivalent octahedral $\mathrm{Fe}^{3+}$ cation sites, i.e. inside the perovskite-like block and on the outer sides of the block, near fluorite like layers $[8,9]$. The relative areas, $A$, of the subspectra show that both mentioned iron sites are approximately equally occupied.

All the magnetoelectric measurements were performed using the dynamic lock-in method described in detail in [16]. The essence of this method is to measure $\mathrm{AC}$ voltage $V_{\text {out }}$ induced between surfaces of the sample as a result of applying a small AC magnetic field $\left(H_{\mathrm{AC}} \ll H_{\mathrm{DC}}\right)$. Moreover, changing the static DC magnetic field one can study the ME coupling at different working points of the sample [16].

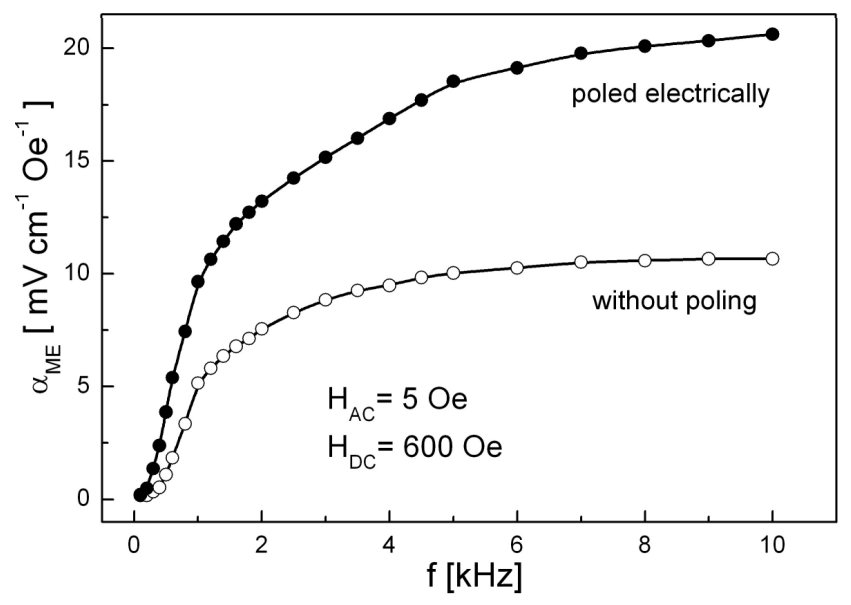

Fig. 4. The dependence of ME coefficient on frequency of $\mathrm{AC}$ magnetic field for $\mathrm{Bi}_{5} \mathrm{Ti}_{3} \mathrm{FeO}_{15}$.

Such a method allows avoiding the problem of charges accumulation at the grain boundaries for polycrystalline samples which significantly affects the output signal in the case of static method [17] and may lead to erroneous conclusions [11]. Figure 4 presents the frequency dependence of $\mathrm{ME}$ coefficient for the $\mathrm{Bi}_{5} \mathrm{Ti}_{3} \mathrm{FeO}_{15}$ sample as well as for the sample subsequently subjected to electrical poling. It may be noted that in the case without initial poling the value of $\alpha_{\mathrm{ME}}$ monotonically increases with increasing frequency of AC field and saturates above $7 \mathrm{kHz}$ reaching maximum value $\alpha_{\mathrm{ME}}=10.7 \mathrm{mV} \mathrm{cm} \mathrm{cm}^{-1} \mathrm{Oe}^{-1}$. Similar course exhibits the curve for poled sample. This time the maximum value $\alpha_{\mathrm{ME}}=20.7 \mathrm{mV} \mathrm{cm}^{-1} \mathrm{Oe}^{-1}$ was registered for the biggest studied frequency $(f=10 \mathrm{kHz})$, and it was almost twice bigger than in the case without the initial poling. So far this is the biggest value reported for the $\mathrm{Bi}_{5} \mathrm{Ti}_{3} \mathrm{FeO}_{15}$ compound. This clearly shows that the proper poling technique may ensure electric dipole reorientation and more effective interaction of magnetic and electric sublattices [17]. The obtained value of the magnetoelectric coefficient for studied material is comparable to the values for the other single-phase ME materials, like $\mathrm{Cr}_{2} \mathrm{O}_{3}$ and $\mathrm{Pb}\left(\mathrm{Fe}_{1 / 2} \mathrm{Nb}_{1 / 2}\right) \mathrm{O}_{3}[18,19]$.

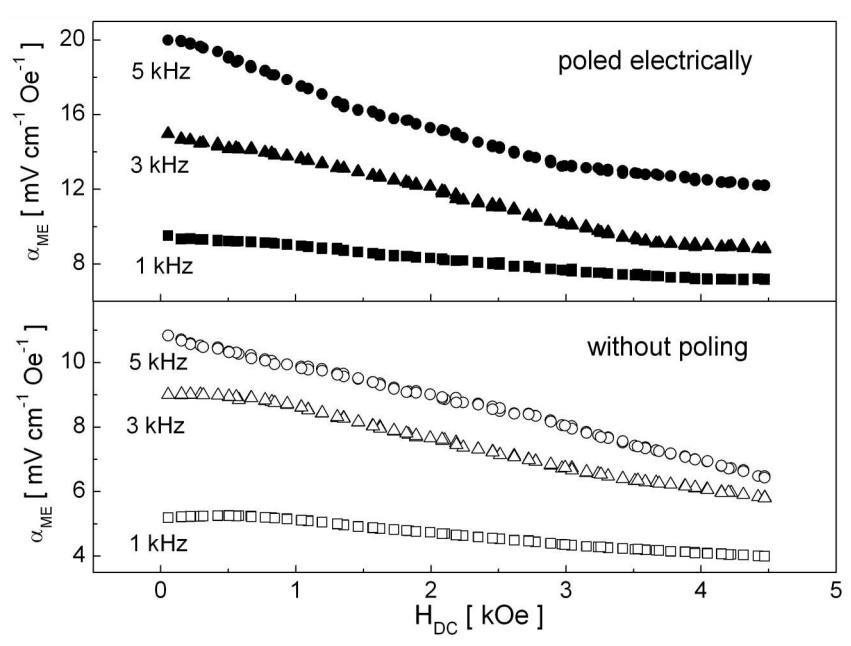

Fig. 5. The dependence of ME coefficient on intensity of DC static field for different frequencies of $\mathrm{AC}$ field for $\mathrm{Bi}_{5} \mathrm{Ti}_{3} \mathrm{FeO}_{15}$.

The dependences of $\alpha_{\mathrm{ME}}$ on static DC magnetic field value for different frequencies of $\mathrm{AC}$ magnetic field are presented in Fig. 5. The value of $\alpha_{\mathrm{ME}}$ slowly decreases with increase of $H_{\mathrm{DC}}$. The measurements were performed along to increasing $H_{\mathrm{DC}}$ value and then back to lower fields. It is relevant to mention that both the curves retrace the same path. There is no trace of hysteresis which was reported in [17] and [13]. The initially poled sample, just like before, shows approximately twice bigger magnetoelectric response than the un-poled one.

\section{Conclusions}

The mixed oxide method was successfully applied to obtain single-phase $\mathrm{Bi}_{5} \mathrm{Ti}_{3} \mathrm{FeO}_{15}$ ceramics. Two doublets used for fitting RT Mössbauer spectra reflect two nonequivalent iron sites in the structure of $\mathrm{Bi}_{5} \mathrm{Ti}_{3} \mathrm{FeO}_{15}$. 
The value of ME coefficient $\alpha_{\mathrm{ME}}$ increases with frequency of $\mathrm{AC}$ magnetic field and decreases with an increase of DC magnetic field. The maximum value of ME coupling coefficient $\alpha_{\mathrm{ME}}=20.7 \mathrm{mV} \mathrm{cm}{ }^{-1} \mathrm{Oe}^{-1}$ was measured for initially poled $\mathrm{Bi}_{5} \mathrm{Ti}_{3} \mathrm{FeO}_{15}$ sample. Proper poling technique applied for multiferroic sample may cause significant increase of ME coupling.

\section{Acknowledgments}

Tomasz Pikula is a participant of the project: "Qualifications for the labour market - employer friendly university", co-financed by European Union from European Social Fund.

\section{References}

[1] M. Fiebig, J. Phys. D Appl. Phys. 38, R123 (2005).

[2] S. Priya, J. Electroceram. 19, 147 (2007).

[3] I. Sosnowska, M. Lowenhaupt, W.I.F. David, R.M. Ibberson, Physica B 180-181, 117 (1992).

[4] G. Catalan, J.F. Scott, Adv. Mater. 21, 2463 (2009).

[5] X.W. Dong, K.F. Wang, J.G. Wan, J.S. Zhu, J.M. Liu, J. Appl. Phys. 103, 094101 (2008).

[6] N.A. Lomanowa, M.I. Morozov, V.L. Ugolkov, V.V. Gusarov, Inorg. Mater. 42, 189 (2006).

[7] N.A. Lomanowa, V.V. Gusarov, Inorg. Mater. 47, 477 (2011).

[8] N.A. Lomanowa, V.G. Semenov, V.V. Panchuk, V.V. Gusarov, J. Alloys Comp. 528, 103 (2012).
[9] E. Jartych, T. Pikula, M. Mazurek, A. LisinskaCzekaj, D. Czekaj, K. Gaska, J. Przewoznik, C. Kapusta, Z. Surowiec, J. Magn. Magn. Mater. 342, 27 (2013).

[10] A. Srinivas, S.V. Suryanarayana, G.S. Kumar, M. Mahesh Kumar, J. Phys. Condens. Matter 11, 3335 (1999).

[11] R.S. Singh, T. Bhimasankaram, G.S. Kumar, S.V. Suryanarayana, Solid State Commun. 91, 567 (1994).

[12] S.V. Suryanarayana, Bull. Mater. Sci. 17, 1259 (1994).

[13] J. Dercz, J. Bartkowska, G. Dercz, P. Stoch, M. Łukasik, Int. J. Thermophys. 34, 567 (2013).

[14] E. Jartych, M. Mazurek, A. Lisinska-Czekaj, D. Czekaj, J. Magn. Magn. Mater. 322, 51 (2010).

[15] A. Lisinska-Czekaj, E. Jartych, M. Mazurek, J. Dzik, D. Czekaj, Ceram. Mater. 62, 126 (2010) (in Polish).

[16] G.V. Duong, R. Groessinger, M. Schoenhart, D. Bueno-Basques, J. Magn. Magn. Mater. 316, 390 (2007).

[17] M. Mahesh Kumar, A. Srinivas, S.V. Suryanarayana, G.S. Kumar, T. Bhimasankaram, Bull. Mater. Sci. 21, 251 (1998).

[18] G. Srinivasan, T. Rasmussen, J. Gallegos, R. Srinivasan, Yu.I. Bokhan, V.M. Laletin, Phys. Rev. B 64, 214408 (2001).

[19] D. Bochenek, P. Guzdek, J. Magn. Magn. Mater. 323, 369 (2011). 